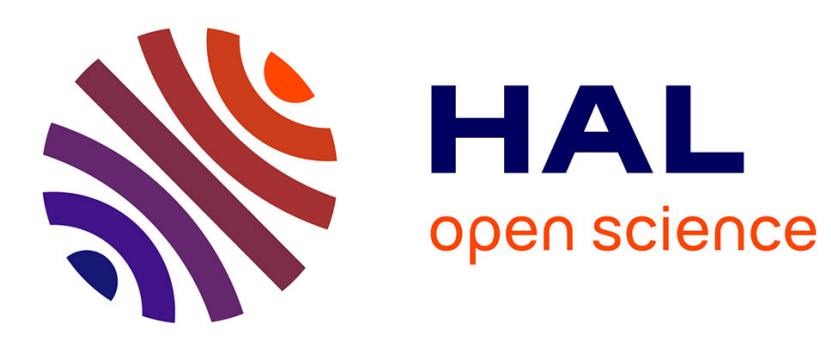

\title{
Elasto-geometrical modeling and calibration of redundantly actuated PKMs
}

\author{
Gaël Ecorchard, Reimund Neugebauer, Patrick Maurine
}

\section{To cite this version:}

Gaël Ecorchard, Reimund Neugebauer, Patrick Maurine. Elasto-geometrical modeling and calibration of redundantly actuated PKMs. Mechanism and Machine Theory, 2010, 45 (5), pp.795-810. 10.1016/j.mechmachtheory.2009.12.008 . hal-00755943

\section{HAL Id: hal-00755943 \\ https://hal.science/hal-00755943}

Submitted on 4 Dec 2012

HAL is a multi-disciplinary open access archive for the deposit and dissemination of scientific research documents, whether they are published or not. The documents may come from teaching and research institutions in France or abroad, or from public or private research centers.
L'archive ouverte pluridisciplinaire HAL, est destinée au dépôt et à la diffusion de documents scientifiques de niveau recherche, publiés ou non, émanant des établissements d'enseignement et de recherche français ou étrangers, des laboratoires publics ou privés. 


\title{
Elasto-geometrical modeling and calibration of redundantly actuated PKMs
}

\author{
Gaël Ecorchard ${ }^{\mathrm{a}, *}$, Reimund Neugebauer ${ }^{\mathrm{b}}$, Patrick Maurine ${ }^{\mathrm{c}}$ \\ ${ }^{a}$ IWP Institute, Chemnitz University of Technology, Reichenhainer Straße 70, \\ 09126, Germany. \\ ${ }^{\mathrm{b}}$ Fraunhofer Institute for Machine Tools and Forming Technology IWU, \\ Reichenhainer Straße 88, 09126 Chemnitz, Germany. \\ ${ }^{\mathrm{c}}$ Laboratory GCGM, Institut National des Sciences Appliquées, 20 Av. des Buttes \\ de Coësmes 35043 Rennes, France.
}

\begin{abstract}
Redundantly actuated Parallel Kinematic Machines (PKMs) offer a number of advantages compared to classical non-redundant PKMs. Particularly, they show a better stiffness thanks to singularity avoidance and they have an improved repeatability due to a better behavior against backlashes. The main problem with the calibration of these machines is that the redundancy leads to some mechanical strains in their structure. This makes it difficult to identify the geometrical errors of their structure without taking into account the effects of the elastic deformations. The main originality of this work is to propose an efficient elasto-geometrical and calibration method that allows the identification of both the geometrical and stiffness parameters of redundantly actuated parallel mechanisms with slender links. The first part of the paper explains the proposed method through its application on a simple redundant planar mechanism. The second part deals with its experimental application to the redundant Scissors Kinematic Machine.
\end{abstract}

Key words: Calibration, Parallel Mechanisms, Redundant Actuation

\section{Introduction}

Redundantly actuated Parallel Kinematic Machines (PKMs) have recently attracted interest of researchers because they allow the reduction of some

\footnotetext{
* Corresponding author. Tel.: +49 37153971410.

Email address: gael.ecorchard@s2001.tu-chemnitz.de (Gaël Ecorchard).
} 
drawbacks of classical, non-redundant PKMs [1]. The presence of one or more redundant actuated chains in the structure allows the avoidance of mechanism singularities [2] [3] and the reduction of joint backlash effects using a control on the internal force directions [4] [5] [6]. Those redundant chains can also be used to perform the autonomous calibration of these mechanisms [7] [8].

Whereas for classical PKMs am insufficient knowledge of the mechanism geometrical properties, such as link length or joint position/orientation, leads exclusively to Cartesian position inaccuracies at the Tool Center Point (TCP) [9], in the case of redundant PKMs, such errors also lead to internal constraints. These mechanical strains in the structure can in turn cause early part wearing and loss of energy in the actuators [5] [10]. These internal constraints make it difficult, not to say impossible, to identify the errors of the geometrical parameters involved in the control model without taking into account the resulting structure elastic deformations. The purpose of this paper is to provide a computation method of the platform situation (position/orientation) of redundantly actuated PKMs and to show how it can be involved in their calibration. By using this method, one can improve the calibration quality of redundant PKMs by carrying out backlash-free measurements while taking into the effect of the internal constraints used to reduce this backlash.

This paper is organized as follows. First, the proposed methodology that is used to derive the elasto-geometrical models of parallel mechanisms with one or more actuated redundant chains is described for planar mechanisms. The development of the method is then illustrated on the Redundant Triglide, a simple redundant planar mechanism. Explanations are then given to show how this modeling method can be easily extended to three dimensional redundant parallel mechanisms. Then, the calibration strategy that has been used to perform the geometrical and stiffness parameter identification of the obtained models is explained. Sensitivity and observability analyses as well as the results of calibration simulations show the efficiency of the proposed approach. The last section deals with the experimental application of the method for the elasto-geometrical modeling and calibration of the redundant Scissors Kinematics Machine developed at the Fraunhofer Institute of Machine Tools and Forming Technology IWU in Chemnitz, Germany.

\section{Elasto-Geometrical Modeling of Redundant PKMs}

\subsection{Method description}

The high dynamics of PKMs suppose low moving masses, i.e., slender elements and light joints [11] [12], which are then subject to elastic deforma- 
tions. For calibration purposes, these elastic deformations that depend on the PKM configuration [13] have to be calculated in order to be compensated. For this purpose, an analytical finite-element modeling using beam elements is proposed to describe redundant PKMs. This approach enables a reduction in the calculation times as well as the number of parameters in comparison to a CAD finite-element method with numerous surfacic or volumic elements. This makes it compatible with calibration issues [14]. However, the calculation of the platform situation for redundant PKMs cannot be achieved in a similar manner as for non-redundant PKMs because it involves an over-determined equation system [15]. The number of loop-closure equations is greater than the platform's degree of freedom. Some authors proposed some purely geometrical methods [16] and some methods based on lumped models [5], [17]. However, the analytical finite-element method allows a more accurate calculation of the platform situation because all possible deformation effects are taken into account [18],[19]. Thus, the final aim of the modeling is to obtain the platform position/orientation $\boldsymbol{X}=\left(\boldsymbol{P}^{\mathrm{T}} \boldsymbol{\phi}^{\mathrm{T}}\right)^{\mathrm{T}}$ under actuation redundancy.

The modeling method is based on the following steps:

(1) Calculation of the platform position/orientation $\boldsymbol{X}_{\mathrm{nr}}=\left(\boldsymbol{P}_{\mathrm{nr}}^{\mathrm{T}} \boldsymbol{\phi}_{\mathrm{nr}}^{\mathrm{T}}\right)^{\mathrm{T}}$ thanks to the forward geometrical model (fgm) of a non-redundant substructure of the redundant mechanism: $\boldsymbol{X}_{\mathrm{nr}}=\operatorname{fgm}\left(\boldsymbol{q}_{\mathrm{nr}}, \boldsymbol{\xi}\right)$, where $\boldsymbol{q}_{\mathrm{nr}}$ is the vector of actuator positions of the non-redundant subsystem and $\boldsymbol{\xi}$, the vector of the geometrical and stiffness parameters.

(2) Calculation of the platform displacements induced by the structure elastic deformations due to its own weight and the applied external forces. This is done through a forward elastic model (fem) $\boldsymbol{\Delta} \boldsymbol{X}_{\mathrm{e}}=\mathrm{fem}(\boldsymbol{q}, \boldsymbol{\xi}, \boldsymbol{F})$, where $\boldsymbol{q}$ is the vector of all actuator positions and $\boldsymbol{F}$ the wrench including the external forces acting on the structure and the structure's own weight.

(3) Calculation of the final platform position/orientation through the resulting forward elasto-geometrical model fegm: $\boldsymbol{X}=\operatorname{fegm}(\boldsymbol{q}, \boldsymbol{\xi}, \boldsymbol{F})=$ $\boldsymbol{X}_{\mathrm{nr}}+\boldsymbol{\Delta} \boldsymbol{X}_{\mathrm{e}}$.

In order to derive the forward elastic model (fem) involved in step (2), the following finite-element approach is used:

- Determination of the stiffness matrices of all links and joints of the mechanism within a local frame attached to each link and joint.

- Calculation of all stiffness matrices into the global reference frame of the structure.

- Mapping and assembly of all the resulting stiffness matrices to derive the global stiffness matrix of the structure.

- Calculation with the global stiffness matrix of the structure of the displacement of all nodes and in particular of the node that corresponds to the TCP.

In next section, the proposed method is detailed for redundant planar parallel 
mechanism and then expanded for three dimensional structures.

\subsection{Elasto-geometrical modeling of redundant planar mechanisms}

In this section the description of the method is presented for planar mechanisms. For this purpose, the values of the geometrical parameters are considered as nominal, that is to say without any errors. As a result, the position and orientation of the platform associated frame is identical and can be calculated with any non-redundant subsystems of the structure. A non-redundant subsystem is defined here as a part of the mechanism that contains as many actuated chains as the platform's degree of freedom and that constitutes a viable mechanism.

\subsubsection{Modeling of the structure links}

The slender links of the planar structures are considered as rods that can be described by planar 2-node beams. For each beam, a local reference frame $\Re_{i j}$ is defined in such a way that its origin is at node $i$, its $\boldsymbol{x}$-axis goes through nodes $i$ and $j$ and its $\boldsymbol{z}$-axis is the same as the $\boldsymbol{z}$-axis of the global frame that is denoted $\Re_{g}$ (Fig. 1). The stiffness of each two-node beam is first expressed in the beam local reference frame $\Re_{i j}$ as [20]:

$$
\mathbf{K}_{i j}=\left(\begin{array}{ll}
\mathbf{K}_{i j}^{a} & \mathbf{K}_{i j}^{b^{\mathrm{T}}} \\
\mathbf{K}_{i j}^{b} & \mathbf{K}_{i j}^{c}
\end{array}\right)
$$

with:

$$
\begin{gathered}
\mathbf{K}_{i j}^{a}=\left(\begin{array}{ccc}
\frac{E S}{L} & 0 & 0 \\
0 & \frac{12 E I_{z}}{L^{3}} & \frac{6 E I_{z}}{L} \\
0 & \frac{6 E I_{z}}{L} & \frac{4 E I_{z}}{L}
\end{array}\right), \quad \mathbf{K}_{i j}^{b}=\left(\begin{array}{ccc}
-\frac{E S}{L} & 0 & 0 \\
0 & -\frac{12 E I_{z}}{L^{3}} & -\frac{6 E I_{z}}{L} \\
0 & \frac{6 E I_{z}}{L} & \frac{2 E I_{z}}{L}
\end{array}\right), \\
\mathbf{K}_{i j}^{c}=\left(\begin{array}{ccc}
\frac{E S}{L} & 0 & 0 \\
0 & \frac{12 E I_{z}}{L^{3}} & -\frac{6 E I_{z}}{L} \\
0 & -\frac{6 E I_{z}}{L} & \frac{4 E I_{z}}{L}
\end{array}\right) .
\end{gathered}
$$

$L$ denotes the element's length, $S$ its cross-section area, $I_{z}$ its quadratic moment along the $\boldsymbol{z}$-axis and $E$ the Young's modulus of its material. For a beam 
with two nodes $i$ and $j$ (Fig. 1) this stiffness matrix gives the relationship between the wrench of external actions $\boldsymbol{F}_{i j}=\left(\boldsymbol{F}_{i}^{\mathrm{T}} \boldsymbol{F}_{j}^{\mathrm{T}}\right)^{\mathrm{T}}$ (forces and moments) applied on its nodes and the resulting linear and rotational displacements, both expressed in the local reference frame $\Re_{i j}$. The wrench of external actions applied on node $i$ is $\boldsymbol{F}_{i}=\left(f_{x, i} f_{y, i} m_{z, i}\right)^{\mathrm{T}}$, where $f_{x, i}$ and $f_{y, i}$ are the forces along $\boldsymbol{x}$ and $\boldsymbol{y}$ and $m_{z, i}$ is the moment around $\boldsymbol{z}$, in the local beam axes. The displacement vector containing the corresponding displacements along $\boldsymbol{x}$ and $\boldsymbol{y}$, and around $\boldsymbol{z}$ is $\boldsymbol{U}_{i}=\left(d_{x, i} d_{y, i} r_{z, i}\right)^{\mathrm{T}}$. We have then:

$$
\boldsymbol{F}_{i j}=\mathbf{K}_{i j} \boldsymbol{U}_{i j}
$$

with: $\boldsymbol{U}_{i j}=\left(\boldsymbol{U}_{i}^{\mathrm{T}} \boldsymbol{U}_{j}^{\mathrm{T}}\right)^{\mathrm{T}}$.

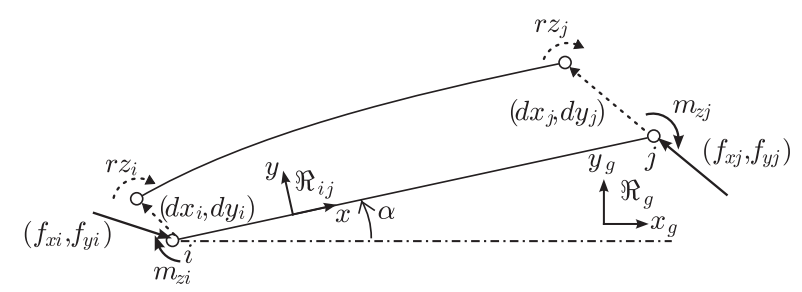

Figure 1. Local wrenches and displacements on an isolated beam.

\subsubsection{Modeling of the structure joints}

The solution that is used here to describe a structure with joints is to consider them as beam elements with coincident nodes. The stiffness of a flexible joint between nodes $k$ and $l$ can be described by the $6 \times 6$ matrix:

$$
\mathbf{K}_{k l}=\left(\begin{array}{cc}
\mathbf{K}_{k l}^{d} & -\mathbf{K}_{k l}^{d} \\
-\mathbf{K}_{k l}^{d} & \mathbf{K}_{k l}^{d}
\end{array}\right)
$$

where $\mathbf{K}_{k l}^{d}=\operatorname{diag}\left(k_{x}, k_{y}, k_{r z}\right)$. The parameters $k_{x}$ and $k_{y}$ stand respectively for the radial stiffness coefficients along $\boldsymbol{x}$ and $\boldsymbol{y}$-axes. $k_{r z}$ is the rotational stiffness coefficient around the $\boldsymbol{z}$-axis. For example, a passive revolute joint of axis $\boldsymbol{z}$ would be described with a beam element with a very small stiffness value for the rotation around the $\boldsymbol{z}$-axis $\left(k_{r z} \approx 0\right)$ and a high stiffness value along the other directions. The small and high stiffness coefficients must be chosen so that they are as far as possible from the other stiffness coefficients and that the numerical accuracy is still valid. A drawback of this solution, called penalty method, is that the setting of these stiffness values is difficult because it must be a compromise between computational instability and results accuracy [21]. Its advantages reside in the ease of implementation and in particular the fact that its associated matrix is positive definite. 


\subsubsection{Mapping and assembly of all stiffness matrices}

During this step, the stiffness contributions of all links and joints are integrated into a global stiffness matrix that describes the whole structure stiffness. For this purpose, the local stiffness matrices of all the elements have to be expressed within the global reference frame $\Re_{g}$ by using the following relation:

$$
\mathbf{K}_{i j}^{g}={ }^{g} \mathbf{T}_{i j}{ }^{\mathrm{T}} \mathbf{K}_{i j}{ }^{g} \mathbf{T}_{i j}
$$

${ }^{g} \mathbf{T}_{i j}=\left(\begin{array}{cc}{ }^{g} \mathbf{R}_{i j} & \mathbf{0}_{3} \\ \mathbf{0}_{3} & { }^{g} \mathbf{R}_{i j}\end{array}\right)$ where ${ }^{g} \mathbf{R}_{i j}$ is the rotation matrix from the local frame $\Re_{i j}$ to the global frame $\Re_{g}$ and $\mathbf{0}_{m}$ is the $m \times m$ null matrix.

If $n$ is the number of nodes used to described the whole structure, the $3 n \times 3 n$ global stiffness matrix $\mathbf{K}^{g}$ is obtained by the addition of the stiffness contribution of each node [22] and the equation system that needs to be solved to calculate all nodal displacements is:

$$
\mathbf{K}^{g} \boldsymbol{U}^{g}=\boldsymbol{F}^{g}
$$

where: $\boldsymbol{U}^{g}=\left(\boldsymbol{U}_{1}^{g \mathrm{~T}} \boldsymbol{U}_{2}^{g \mathrm{~T}} \ldots \boldsymbol{U}_{n}^{g \mathrm{~T}}\right)^{\mathrm{T}}$ and $\boldsymbol{F}^{g}=\left(\boldsymbol{F}_{1}^{g \mathrm{~T}} \boldsymbol{F}_{2}^{g \mathrm{~T}} \ldots \boldsymbol{F}_{n}^{g \mathrm{~T}}\right)^{\mathrm{T}} . \boldsymbol{F}_{i}^{g}$ and $\boldsymbol{U}_{i}^{g}$ correspond to $\boldsymbol{F}_{i}$ and $\boldsymbol{U}_{i}$ expressed in $\Re_{g}$.

It is to be noted that the elastic deformations that are induced by the structure's own weight are considered by reporting the weight of each link to its two associated nodes and then by merging the resulting equivalent efforts to the vector of external actions $\boldsymbol{F}^{g}$.

\subsubsection{Modeling of links that are connected to the base or the ground}

The proposed method requires differenciating between the modeling of the passive and actuated links that connect the PKM structure to its base or to the ground.

- For the modeling of the passive links, some boundary conditions must be defined in order to express the fact that those links are free to move in some directions and blocked in others. This is done by removing the rows and columns of the stiffness matrix $\mathbf{K}^{g}$ related to the nodes of those links where a null displacement condition is imposed. This then leads to the reduced stiffness matrix $\mathbf{K}^{r}$, the vectors $\boldsymbol{F}^{r}$ and $\boldsymbol{U}^{r}$. To illustrate this, one can consider the structure of Fig. 2 for which the length of the actuated redundant leg varies thanks to the actuator $q_{3}$. As one can see, the node 8 that describes the passive joint between the redundant leg and the base can turn freely around the $\boldsymbol{z}$-axis but its position is blocked. To relate those 
conditions, the equations associated to the translational displacements of node 8 along $\boldsymbol{x}$ and $\boldsymbol{y}$ are removed from the equation system (7).

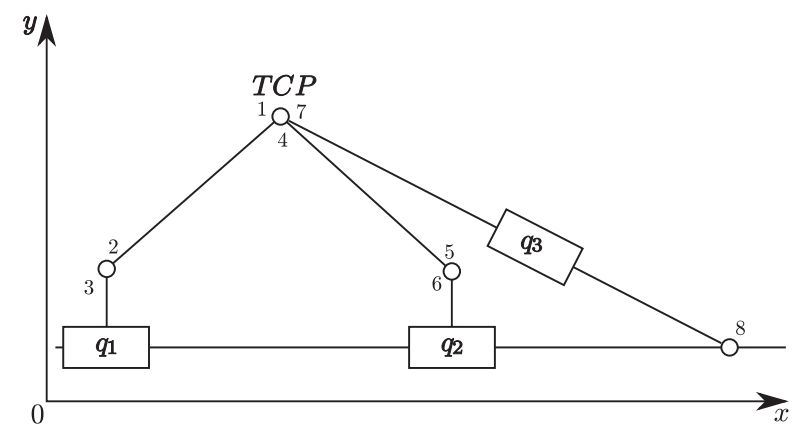

Figure 2. Biglide with an actuated redundant branch.

- In a context of calibration, the modeling of the structure's actuated links that are connected to the base or to the ground is done by considering that the elements related to them are fixed to the base (as for example the carriers $q_{1}$ and $q_{2}$ on the structure of Fig. 2). However those elements can still be considered as flexible and thus they will be described by using a 1-node beam element. This beam is connected at one end to the structure through the node and it is at its other end fixed to the base. Its stiffness is then given by the $3 \times 3$ matrix:

$$
\mathbf{K}_{i}=\mathbf{K}_{i j}^{c}
$$

where $\mathbf{K}_{i j}^{c}$ is the matrix defined by (3) and $i$ the node associated to the element. Since 1-node beam elements are used in the modeling for the links connected to the base, no boundary conditions need to be defined, so that the reduced stiffness matrix is $\mathbf{K}^{r}=\mathbf{K}^{g}, \boldsymbol{F}^{r}=\boldsymbol{F}^{g}$ and $\boldsymbol{U}^{r}=\boldsymbol{U}^{g}$.

After the application of boundary conditions depending of the type of links between the base and the mechanism, the equation system that has to be solved to obtain the nodal displacements of the whole structure is

$$
\mathbf{K}^{r} \boldsymbol{U}^{r}=\boldsymbol{F}^{r}
$$

\subsubsection{Calculation of the elastic displacements}

Equation system (9) has a unique solution because the matrix $\mathbf{K}^{r}$ is symmetric positive definite. It can be calculated according to the relation $\boldsymbol{U}^{r}=\mathbf{K}^{r-1} \boldsymbol{F}^{r}$ by inverting the matrix $\mathbf{K}^{r}$ but it can be more efficiently obtained by solving the system through its singular value decomposition (SVD) [23]. The elastic translational and rotational displacements of the frame associated to the platform is the part of $\boldsymbol{U}^{r}$ that corresponds to the elastic displacements of the node $t$ associated to it.

$$
\Delta \boldsymbol{X}_{\mathbf{e}}=\boldsymbol{U}_{t}^{r}
$$


The final platform position/orientation is then obtained through the elastogeometrical model as

$$
\boldsymbol{X}=\operatorname{fgm}\left(\boldsymbol{q}_{\mathrm{nr}}, \boldsymbol{\xi}\right)+\boldsymbol{\Delta} \boldsymbol{X}_{\mathbf{e}}=\operatorname{fegm}(\boldsymbol{q}, \boldsymbol{\xi}, \boldsymbol{F}),
$$

where $\boldsymbol{F}$ is the wrench of external forces that can possibly be applied on the structure nodes.

\subsection{Illustration of the method with the Redundant Triglide structure}

\subsubsection{Description of the Redundant Triglide}

To illustrate the method that is proposed for the elasto-geometrical modeling of redundant PKM, it is applied to a simple planar mechanism with an actuated redundant branch. This planar two-degree-of-freedom mechanism that will be called Redundant Triglide in the following is described in Fig. 3 with exaggerated geometrical parameter errors.

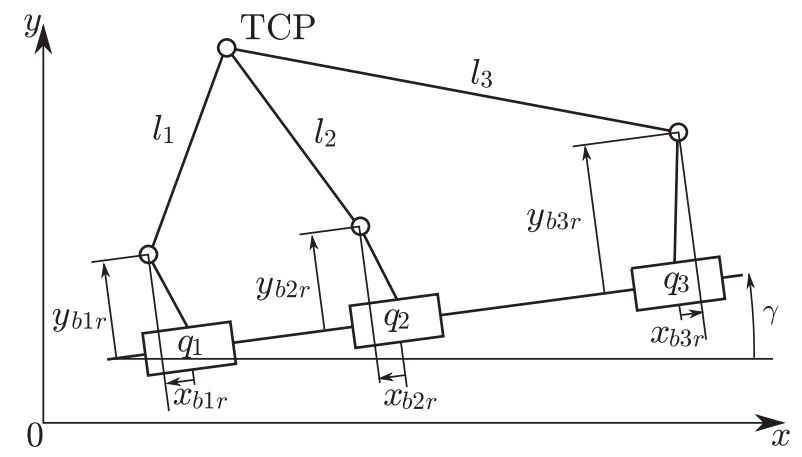

Figure 3. Schematic representation of the Redundant Triglide

Its moving platform is considered as a point (TCP). The axes of the three prismatic joints are assumed to be colinear since they are mounted on the same guide. The actuators $q_{1}$ and $q_{2}$ are considered in the following as part of the non-redundant subsystem. Therefore, actuator $q_{3}$ is the redundant actuator. The loop-closure equation of $\operatorname{rod} l_{i}$ is:

$$
f_{i}=\left(x-q_{i}-x_{b i r} \cos \gamma+y_{b i r} \sin \gamma\right)^{2}+\left(y-q_{i}-x_{b i r} \sin \gamma-y_{b i r} \cos \gamma\right)^{2}-l_{i}^{2}
$$

where $\left(\begin{array}{ll}x & y\end{array}\right)$ are the co-ordinates of the TCP and $q_{i}$ the position of actuator $i$. The parameters $x_{b i r}, y_{b i r}, l_{i}$, and $\gamma$ are described in Fig. 3 and the nominal values that will be used for simulations are given in Table 1 where the letter $r$ used in the parameter name indices stands for relative reference frame. The TCP position of the Redundant Triglide is first calculated through the forward geometrical model $\boldsymbol{X}_{\mathrm{nr}}=\operatorname{fgm}\left(\boldsymbol{q}_{\mathrm{nr}}, \boldsymbol{\xi}\right)$ with $\boldsymbol{q}_{\mathrm{nr}}=\left(q_{1}, q_{2}\right)^{\mathrm{T}}$. It is obtained as the intersection of the two circles centered on the joint of the carriers $q_{1}$ and $q_{2}$ and which radii are $l_{1}$ and $l_{2}$ respectively. 
Table 1

Nominal parameter values ( $\mathrm{mm}$ and $\mathrm{deg}$ ).

\begin{tabular}{|c|c|c|c|c|c|c|c|c|c|}
\hline$l_{1}$ & $l_{2}$ & $l_{3}$ & $x_{b 1 r}$ & $y_{b 1 r}$ & $x_{b 2 r}$ & $y_{b 2 r}$ & $x_{b 3 r}$ & $y_{b 3 r}$ & $\gamma$ \\
\hline 300 & 300 & 600 & 0 & 100 & 0 & 100 & 0 & 150 & 0 \\
\hline
\end{tabular}

\subsubsection{Modeling of Triglide links and joints}

The model of the Redundant Triglide structure and the numbering of the corresponding nodes are described in Fig. 4. As it can be viewed as a 2D mechanism, only planar elements (beams) are involved for its elastic modeling. A number of 9 nodes is used to describe its structure. The link (for example between nodes 1 and 2) and the carriers (for example the element linked to node 3) are represented by one beam element each as explained in Section 2.2.4. These elements are linked together by 5 revolute joints to which stiffness coefficients are assigned.

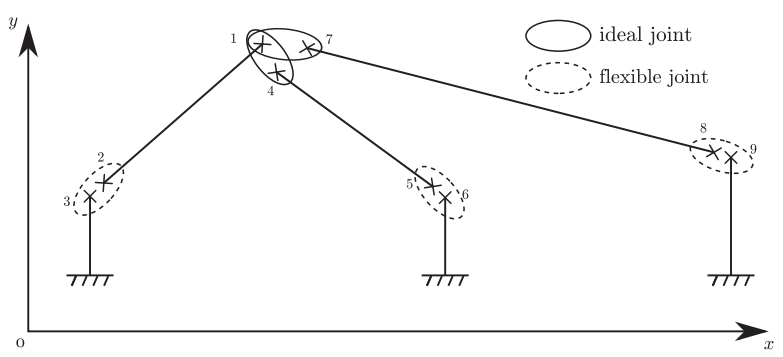

Figure 4. Beam-element model of the Triglide mechanism.

The axial rotational rigidity coefficients of all passive revolute joints is considered to be small enough to have no influence on the deformations of the Triglide structure. Therefore, the revolute joints are only stressed in the radial directions and the stiffness of the two joints associated to one link can be combined into only one stiffness coefficient assigned to one of the two joints. As a result, the two joints that correspond to the TCP (linking nodes 1 and 4 on one hand and nodes 1 and 7 on the other hand) are modeled as ideal revolute joints, whereas the revolute joints between the carriers and the rods have a given radial stiffness.

\subsubsection{Mapping and assembly of the link and joint stiffness matrices}

In order to perform the assembly of the global stiffness matrix, the local stiffness matrices of all the links and joints have to be calculated within the global reference frame $\Re_{g}$. For example, the stiffness matrix of the beam element between nodes 1 and 2 in $\Re_{g}$ is obtained as follows:

$$
\mathbf{K}_{12}^{g}={ }^{g} \mathbf{T}_{12}{ }^{\mathrm{T}} \mathbf{K}_{12}{ }^{g} \mathbf{T}_{12} .
$$


The $27 \times 27$ global stiffness matrix of the structure $\mathbf{K}^{g}$ is then obtained by the mapping of the stiffness contribution of each node.

$$
\mathbf{K}^{g}=\left(\begin{array}{cc}
\mathbf{K}^{1} & \mathbf{K}^{2^{\mathrm{T}}} \\
\mathbf{K}^{2} & \mathbf{K}^{3}
\end{array}\right)
$$

with:

$$
\begin{aligned}
& \mathbf{K}^{1}=\left(\begin{array}{cccc}
\mathbf{K}_{12}^{a g}+\mathbf{K}_{14}^{d g}+\mathbf{K}_{17}^{d g} & \mathbf{K}_{12}^{b g^{\mathrm{T}}} & \mathbf{0}_{3} & -\mathbf{K}_{14}^{d g} \\
\mathbf{K}_{12}^{b g} & \mathbf{K}_{12}^{c g}+\mathbf{K}_{23}^{d g} & -\mathbf{K}_{23}^{d g} & \mathbf{0}_{3} \\
\mathbf{0}_{3} & -\mathbf{K}_{23}^{d g} & \mathbf{K}_{23}^{d g}+\mathbf{K}_{3}^{a g} & \mathbf{0}_{3} \\
-\mathbf{K}_{14}^{d g} & \mathbf{0}_{3} & \mathbf{0}_{3} & \mathbf{K}_{14}^{d g}+\mathbf{K}_{45}^{a g}
\end{array}\right) ; \\
& \mathbf{K}^{2}=\left(\begin{array}{cccc}
\mathbf{0}_{3} & \mathbf{0}_{3} & \mathbf{0}_{3} & \mathbf{K}_{45}^{b g} \\
\mathbf{0}_{3} & \mathbf{0}_{3} & \mathbf{0}_{3} & \mathbf{0}_{3} \\
-\mathbf{K}_{17}^{c g} & \mathbf{0}_{3} & \mathbf{0}_{3} & \mathbf{0}_{3} \\
\mathbf{0}_{3} & \mathbf{0}_{3} & \mathbf{0}_{3} & 0_{3} \\
\mathbf{0}_{3} & \mathbf{0}_{3} & \mathbf{0}_{3} & \mathbf{0}_{3}
\end{array}\right) \\
& \mathbf{K}^{3}=\left(\begin{array}{ccccc}
\mathbf{K}_{45}^{c g}+\mathbf{K}_{56}^{d g} & -\mathbf{K}_{56}^{d g} & \mathbf{0}_{3} & \mathbf{0}_{3} & \mathbf{0}_{3} \\
-\mathbf{K}_{56}^{d g} & \mathbf{K}_{56}^{d g}+\mathbf{K}_{6}^{a g} & \mathbf{0}_{3} & \mathbf{0}_{3} & \mathbf{0}_{3} \\
\mathbf{0}_{3} & \mathbf{0}_{3} & \mathbf{K}_{17}^{d g}+\mathbf{K}_{78}^{a g} & \mathbf{K}_{78}^{b g^{\mathrm{T}}} & \mathbf{0}_{3} \\
\mathbf{0}_{3} & \mathbf{0}_{3} & \mathbf{K}_{78}^{b g} & \mathbf{K}_{78}^{c g}+\mathbf{K}_{89}^{d g} & -\mathbf{K}_{89}^{d g} \\
\mathbf{0}_{3} & \mathbf{0}_{3} & \mathbf{0}_{3} & -\mathbf{K}_{89}^{d g} & \mathbf{K}_{89}^{d g}
\end{array}\right) .
\end{aligned}
$$

$\mathbf{K}_{i j}^{a g}, \mathbf{K}_{i j}^{b g}, \mathbf{K}_{i j}^{c g}$, and $\mathbf{K}_{i j}^{d g}$ are the stiffness submatrices for the element between nodes $i$ and $j$ defined in sections 2.2.1 and 2.2.2 and that are rewritten in the global reference frame $\Re_{g}$.

\subsubsection{Modeling of the links connected to the base}

All links connecting the mechanism structure and its base are actuated. As explained, they will be considered to be fixed to the base for the calibration. The three carriers are described by 1-node beam elements which means that no boundary conditions need to be applied for the definition of $\mathbf{K}^{r}$. 


\subsubsection{Calculation of all nodal displacements}

The calculation of the nodal displacements that are gathered in $\boldsymbol{U}^{g}$ is done by solving equation (7). The TCP displacement due to elastic deformations is

$$
\Delta \boldsymbol{X}_{\mathbf{e}}=\left(\begin{array}{l}
d_{x_{1}} \\
d_{y_{1}}
\end{array}\right)
$$

where $d_{x_{1}}$ and $d_{y_{1}}$ are the displacements of node 1 in $\boldsymbol{x}$ and $\boldsymbol{y}$-directions respectively, i.e., the first two components of $\boldsymbol{U}^{g}$. The node rotational displacement around the $\boldsymbol{z}$-axis is not considered.

The final TCP position is calculated through the resulting forward elastogeometrical model $\boldsymbol{X}=\operatorname{fgm}\left(\boldsymbol{q}_{\mathrm{nr}}, \boldsymbol{\xi}\right)+\boldsymbol{\Delta} \boldsymbol{X}_{\mathbf{e}}$.

\subsection{Generalization of the proposed method to three-dimensional mechanisms}

In this section, the extension of the elasto-geometrical modeling method is presented for three-dimensional mechanisms. For this purpose, the nodal force and displacement vectors are modified to consider the six degree-of-freedom as follows:

$$
\boldsymbol{F}_{i}=\left(f_{x, i} f_{y, i} f_{z, i} m_{x, i} m_{y, i} m_{z, i}\right)^{\mathrm{T}} \quad \boldsymbol{U}_{i}=\left(d_{x, i} d_{y, i} d_{z, i} r_{x, i} r_{y, i} r_{z, i}\right)^{\mathrm{T}}
$$

where $f_{z, i}$ is the force along $\boldsymbol{z}$ and $m_{x, i}$ and $m_{y, i}$ are the moments around $\boldsymbol{x}$ and $\boldsymbol{y}$, in the local beam axis. The displacements are $d_{x, i}, d_{y, i}, d_{z, i}$, for the local displacements along $\boldsymbol{x}, \boldsymbol{y}$ and $\boldsymbol{z}$ respectively, and $r_{x, i}, r_{y, i}$ and $r_{z, i}$, for the local rotations around these same axes. For the modeling of the structure bodies, the $12 \times 12$ stiffness matrix that is used to describe a 2-node link between the nodes $i$ and $j$ is:

$$
\mathbf{K}_{i j}=\left(\begin{array}{cc}
\mathbf{K}_{i j}^{a} & \mathbf{K}_{i j}^{b^{\mathrm{T}}} \\
\mathbf{K}_{i j}^{b} & \mathbf{K}_{i j}^{c}
\end{array}\right)
$$

with:

$$
\mathbf{K}_{i j}^{a}=\left(\begin{array}{cccccc}
\frac{E S}{L} & 0 & 0 & 0 & 0 & 0 \\
0 & \frac{12 E I_{z}}{L^{3}} & 0 & 0 & 0 & \frac{6 E I z}{L^{2}} \\
0 & 0 & \frac{12 E I_{y}}{L^{3}} & 0 & -\frac{6 E I_{y}}{L^{2}} & 0 \\
0 & 0 & 0 & \frac{G J}{L} & 0 & 0 \\
0 & 0 & -\frac{6 E I_{y}}{L^{2}} & 0 & \frac{4 E I_{y}}{L} & 0 \\
0 & \frac{6 E I_{z}}{L^{2}} & 0 & 0 & 0 & \frac{4 E I z}{L}
\end{array}\right)
$$




$$
\begin{aligned}
\mathbf{K}_{i j}^{b}=\left(\begin{array}{cccccc}
-\frac{E S}{L} & 0 & 0 & 0 & 0 & 0 \\
0 & -\frac{12 E I_{z}}{L^{3}} & 0 & 0 & 0 & -\frac{6 E I z}{L^{2}} \\
0 & 0 & -\frac{12 E I_{y}}{L^{3}} & 0 & \frac{6 E I_{y}}{L^{2}} & 0 \\
0 & 0 & 0 & -\frac{G J}{L} & 0 & 0 \\
0 & 0 & -\frac{6 E I_{y}}{L^{2}} & 0 & \frac{2 E I_{y}}{L} & 0 \\
0 & \frac{6 E I_{z}}{L^{2}} & 0 & 0 & 0 & \frac{2 E I z}{L}
\end{array}\right) \\
\mathbf{K}_{i j}^{c}=\left(\begin{array}{cccccc}
\frac{E S}{L} & 0 & 0 & 0 & 0 & 0 \\
0 & \frac{12 E I_{z}}{L^{3}} & 0 & 0 & 0 & -\frac{6 E I z}{L^{2}} \\
0 & 0 & \frac{12 E I_{y}}{L^{3}} & 0-\frac{6 E I_{y}}{L^{2}} & 0 \\
0 & 0 & 0 & \frac{G J}{L} & 0 & 0 \\
0 & 0 & \frac{6 E I_{y}}{L^{2}} & 0 & \frac{4 E I_{y}}{L} & 0 \\
0 & -\frac{6 E I_{z}}{L^{2}} & 0 & 0 & 0 & \frac{4 E I z}{L}
\end{array}\right)
\end{aligned}
$$

The joint stiffness is described by a matrix $\mathbf{K}_{k l}$ as in (5). The difference with the modeling of planar mechanisms is that the submatrix $\mathbf{K}_{k l}^{d}$ must be extended as $\mathbf{K}_{k l}^{d}=\operatorname{diag}\left(k_{x}, k_{y}, k_{z}, k_{r x}, k_{r y}, k_{r z}\right)$. The parameters $k_{z}, k_{r x}$ and $k_{r y}$ stand for the radial stiffness coefficient along $\boldsymbol{z}$, the rotational stiffness coefficient around $\boldsymbol{x}$ and the rotational stiffness coefficient around $\boldsymbol{y}$, respectively. Finally, to obtain the nodal displacements of the entire structure, the series of equations (4) to (9) that have been used in the case of planar mechanisms are applied again with $\boldsymbol{F}_{i}, \boldsymbol{U}_{i}$ and $\mathbf{K}_{i j}$ as previously described.

\section{Elasto-geometrical calibration of redundant PKMs}

In the first part of this section the elasto-geometrical modeling method that has been proposed previously is modified to be used for calibration. This leads to the error model that will be involved for the identification of both geometrical and stiffness parameters of the PKM structure. In the second part of the section, the whole calibration methodology will be illustrated by using again the Redundant Triglide. In order to evaluate the influence of the redundancy onto the PKM calibration, the calibration of a non-redundant sub-structure of the Triglide - that is to say without the redundant branch is also considered. 
The calibration method is described in Fig. 5. In this figure $\boldsymbol{\xi}_{\text {nom }}$ are the nominal parameters, $\boldsymbol{q}_{\text {set }}$ are the actuator set values, $\Delta \boldsymbol{X}$ is the vector of measurement errors obtained by the difference between $\boldsymbol{X}_{\text {mod }}$ and $\boldsymbol{X}_{\text {meas }}, \mathbf{J}^{*}$ is the pseudo-inverse of the Jacobian matrix of the calibration and $\boldsymbol{\xi}_{\text {cal }}$ is the vector of calibrated parameters.

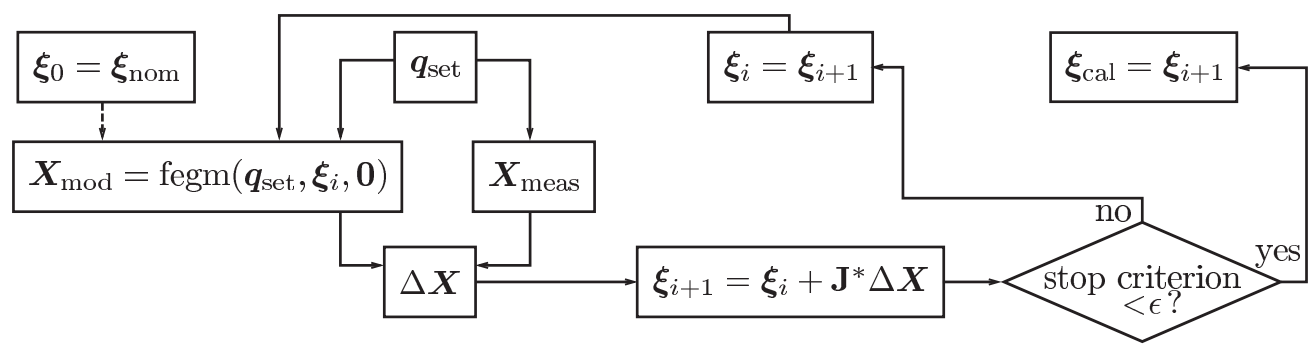

Figure 5. Calibration method with use of the forward elasto-geometrical model.

\subsection{Error model for the calibration of redundant PKMs}

In order to derive the calibration error model, the situation of the PKM platform has to be calculated with both nominal and real parameters, respectively $\boldsymbol{\xi}_{\text {nom }}$ and $\boldsymbol{\xi}=\boldsymbol{\xi}_{\text {nom }}+\Delta \boldsymbol{\xi}$. However, the consideration of some geometrical errors $\Delta \boldsymbol{\xi}$ leads to the fact that the nodes of some joints involved in the elastic modeling are not coincident anymore and the proposed elasto-geometrical method has to be modified.

To illustrate this problem with the Redundant Triglide, we first consider that the position of all actuators is calculated for a given TCP position with the nominal geometrical parameters. Then, an error $\Delta l_{3}$ is introduced in the length of the third rod $l_{3}$. As one can see in Fig. 6, the loop-closure equation cannot be satisfied anymore or in other words, the joint nodes are not coincident. As a result, a solution has to be found to calculate the structure's nodal displacements for PKM with some geometrical errors with the FE analysis.

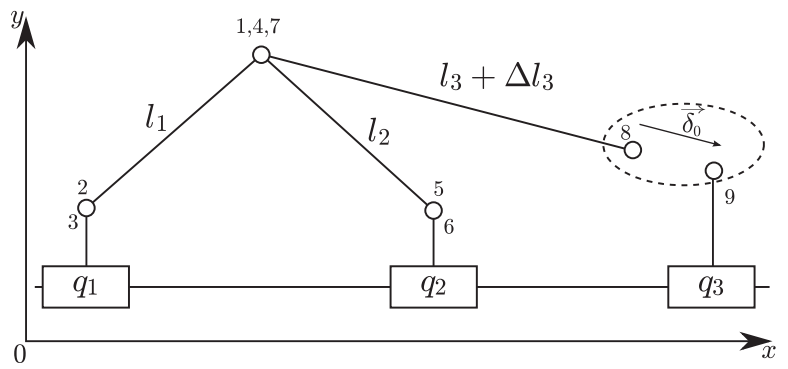

Figure 6. Non-coincidence of node joints in case of geometrical errors.

As explained previously, the elastic joints are described by beam elements whose length is null and for which the stiffness matrix is diagonal. At this point, two cases have to be considered: 
- If no geometrical error affects the mechanism, the nodes of the joints are coincident and the distance between them after the application of external forces will be calculated through the resolution of (7). Figures 7(a) and 7(b) give an example: the distance between the two nodes after application of a force $f_{y}$ in the $\boldsymbol{y}$-direction will be $\delta=k_{y} f_{y}$.

- If some geometrical errors exist, the distance between the two nodes of some joints is non-null before application of the external forces and they will behave as beam elements with an initial length $\delta_{0}$. Their node displacements calculated through the resolution of (7) will not fit with reality. As one can see in Fig. 8(a), when the nodes are separated by a distance $\delta_{0}$ during the assembly step, their final distance after calculation of nodal displacements will be $\delta^{\prime}=\delta_{0}+k_{y} f_{y}$ (Fig. 8(b)).

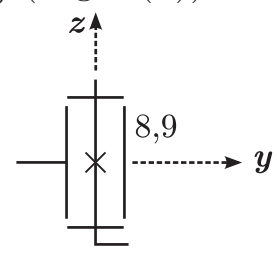

(a)At assembly step.

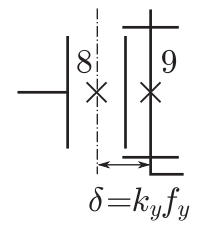

(b)After resolution.

Figure 7. Deformation of flexible joints with initial coincident nodes.

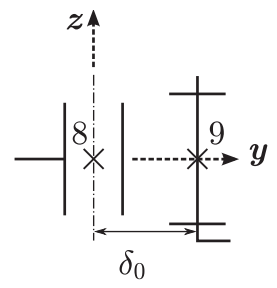

(a)At assembly step.

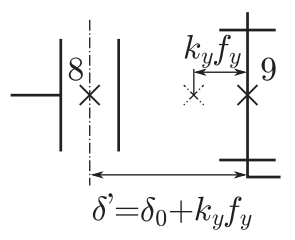

(b)After resolution.

Figure 8. Deformation of flexible joints with non-coincident nodes.

\subsubsection{Method of joint internal forces}

The proposed solution to solve the problem of non-coincident joints consists in the following steps:

(1) Calculating the platform position/orientation with the forward geometrical model $\boldsymbol{X}_{\mathrm{nr}}=\operatorname{fgm}\left(\boldsymbol{q}_{\mathrm{nr}}, \boldsymbol{\xi}\right)$ of any non-redundant substructure of the machine where $\boldsymbol{q}_{\mathrm{nr}}$ is the actuator position vector of the non-redundant subsystem.

(2) Calculating the position of the nodes of the redundant link(s). The nominal geometrical parameters $\boldsymbol{\xi}_{\text {nom }}$ have to be used for this calculation and the distance between two nodes of a joint has to be minimal.

(3) Modifying the right hand side of the equation system (7) by merging into $\boldsymbol{F}^{r}$ the vector of initial external wrenches that have to be applied to cancel the gap existing between the non-coincident nodes of elastic joints. 
(4) Assembling the stiffness matrix and solving (7) by using the modified right hand side.

This proposed solution is illustrated with the Triglide (Fig. 9). For step 2, the position of node 8 is given by:

$$
\boldsymbol{X}_{8}=\left(\begin{array}{l}
x-\left(l_{3}+\Delta l_{3}\right) \cos \alpha_{3} \\
y-\left(l_{3}+\Delta l_{3}\right) \sin \alpha_{3}
\end{array}\right)
$$

where $\alpha_{3}$ is the rod angle with the $\boldsymbol{x}$-axis, calculated with nodes 4 and 9 .

For step 3, the joint stiffness is given by (5) and, since the distance $\delta_{0}$ between the two nodes 8 and 9 is non null (Fig. 9), an internal force proportional to the distance has to be applied and added to the right-hand side of the equation system as follows:

$$
\boldsymbol{F}_{89}^{\prime}=\boldsymbol{F}_{89}+\boldsymbol{K}_{89}\left(\begin{array}{c}
\boldsymbol{X}_{9}-\boldsymbol{X}_{8} \\
0 \\
\boldsymbol{X}_{8}-\boldsymbol{X}_{9} \\
0
\end{array}\right)
$$

where $\boldsymbol{X}_{i}$ is the absolute co-ordinate vector of node $i$.

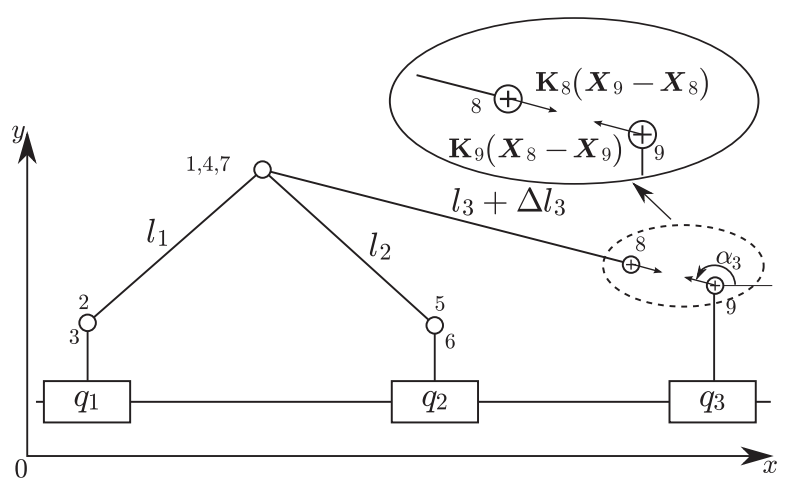

Figure 9. Solving the problem of non-coincident joints by considering internal forces.

For step 4, the system (7) is solved to derive all nodal displacements and then the position of the Triglide's TCP (Fig. 10).

\subsection{Global Jacobian matrix for the calibration}

In order to perform the calibration, the local Jacobian matrix $\mathbf{J}_{i}$ that gives for a configuration $i$ the relationship between the variations of the geometri$\mathrm{cal} /$ stiffness parameters and the variations of the PKM platform situation is 


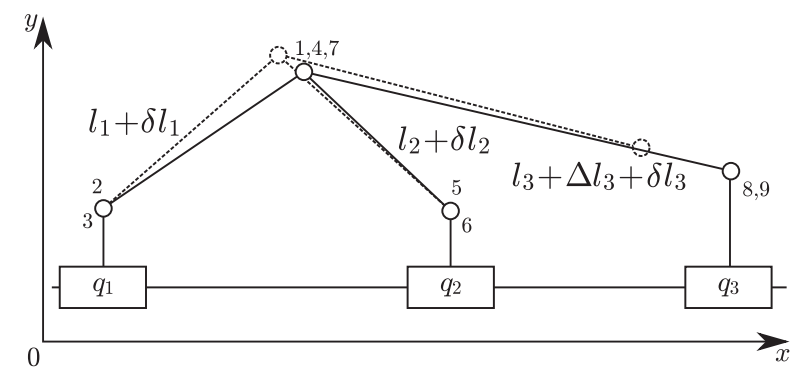

Figure 10. Solution of the forward elasto-geometrical model.

calculated as follows:

$$
\mathbf{J}_{i}=\left(\begin{array}{cccc}
\frac{\partial P_{x}}{\partial \xi_{1}} & \frac{\partial P_{x}}{\partial \xi_{2}} & \cdots & \frac{\partial P_{x}}{\partial \xi_{p}} \\
\frac{\partial P_{y}}{\partial \xi_{1}} & \frac{\partial P_{y}}{\partial \xi_{2}} & \cdots & \frac{\partial P_{y}}{\partial \xi_{p}} \\
\cdots & \cdots & \cdots & \cdots \\
\frac{\partial \phi_{z}}{\partial \xi_{1}} & \frac{\partial \phi_{z}}{\partial \xi_{2}} & \cdots & \frac{\partial \phi_{z}}{\partial \xi_{p}}
\end{array}\right) .
$$

$\frac{\partial P_{x}}{\partial \xi_{i}}, \frac{\partial P_{y}}{\partial \xi_{i}}, \cdots, \frac{\partial \phi_{z}}{\partial \xi_{i}}$ are the partial derivatives of the platform position/orientation with respect to the parameter $\xi_{i},(i=1, \ldots, p)$ where $p$ is the number of parameters to be identified. Since the finite-element method that is used to derive this forward elasto-geometrical model (fegm) requires a numerical solution of the equation system (7), those partial derivatives are calculated as a finite-difference of (11). Then, the $6 m \times p$ global Jacobian matrix $\mathbf{J}$ that will be involved further for the sensitivity and observability analyses is obtained as the concatenation of the local Jacobian matrices $\mathbf{J}_{i}$ calculated for the $m$ configurations of measurement.

\subsection{Sensitivity and observability analyses}

The aim of the sensitivity analysis is to evaluate the platform position / orientation variations as a function of each geometrical/stiffness parameter variation. The range in which each of those parameters can vary can be set based on considerations related to the machine part manufacturing and assembly. Then, in order to compare the effects of parameters having different units and orders of magnitude, the global Jacobian matrix is normalized [24]. For this purpose, let us define $\Delta \xi_{i}=\xi_{i}^{\max }-\xi_{i}^{\text {nom }}$, the variation range of parameter $i$ where $\xi_{i}^{\max }$ and $\xi_{i}^{\text {nom }}$ are respectively the maximal and nominal values of the parameter. The $p \times p$ matrix $\mathbf{H}^{s}=\operatorname{diag}\left(\Delta \xi_{1}, \Delta \xi_{2}, \cdots, \Delta \xi_{p}\right)$ is then built to normalize $\mathbf{J}$ according to $\mathbf{J}^{s}=\mathbf{J} \mathbf{H}^{s}$. Then, for each measurement configuration $i(i=1, \ldots, m)$ and for each parameter $\mathrm{j}(j=1, \ldots, p)$, let us define:

$$
\begin{array}{lll}
\sigma_{P_{x, i, j}}=\mathbf{J}^{s}{ }_{6 \times i-5, j} & \sigma_{P_{y, i, j}}=\mathbf{J}^{s}{ }_{6 \times i-4, j} & \sigma_{P_{z, i, j}}=\mathbf{J}^{s}{ }_{6 \times i-3, j} \\
\sigma_{\phi_{x, i, j}}=\mathbf{J}^{s}{ }_{6 \times i-2, j} & \sigma_{\phi_{y, i, j}}=\mathbf{J}^{s}{ }_{6 \times i-1, j} & \sigma_{\phi_{z, i, j}}=\mathbf{J}_{6 \times i, j}^{s}
\end{array}
$$


$\left(\sigma_{P_{x, i, j}}, \sigma_{P_{y, i, j}}, \sigma_{P_{z, i, j}}\right)$ and $\left(\sigma_{\phi_{x, i, j}}, \sigma_{\phi_{y, i, j}}, \sigma_{\phi_{z, i, j}}\right)$ correspond respectively to the variations on the platform position and orientation along the $\boldsymbol{x}, \boldsymbol{y}$ and $\boldsymbol{z}$ directions for measurement point $i$ as a consequence to a variation $\delta_{j}$ on parameter $j$. This enables defining the two local indexes $\sigma_{P_{i, j}}$ and $\sigma_{\phi_{i, j}}$ giving respectively the sensitivity of the platform position and orientation to the variation of $\xi_{j}$ :

$$
\begin{aligned}
\sigma_{P_{i, j}} & =\sqrt{\sigma_{P_{x, i, j}}^{2}+\sigma_{P_{y, i, j}}^{2}+\sigma_{P_{z, i, j}}^{2}} \\
\sigma_{\phi_{i, j}} & =\sqrt{\sigma_{\phi_{x, i, j}}^{2}+\sigma_{\phi_{y, i, j}}^{2}+\sigma_{\phi_{z, i, j}}^{2}}
\end{aligned}
$$

By using the local indexes calculated for PKM configurations uniformly placed in the workspace, the global sensitivity indexes $\sigma_{P_{j}}$ and $\sigma_{\phi_{j}}$ are then derived as follows:

$$
\sigma_{P_{j}}=\frac{\sum_{i=1}^{m} \sigma_{P_{i, j}}}{m} \quad \sigma_{\phi_{j}}=\frac{\sum_{i=1}^{m} \sigma_{\phi_{i, j}}}{m}
$$

The study of those two global indexes allows one to select among the geometrical/stiffness parameters $\xi_{j}$ those that take priority in being identified. Their observability is verified through the SVD of global Jacobian matrix $\mathbf{J}^{s}$ from which only the columns of the selected parameters are kept. All details of this observability analysis are available in [25][26].

Let us illustrate this with the Triglide for which the workspace corresponds to a movement of the TCP from $y=160$ to $390 \mathrm{~mm}$ since a movement along the $\boldsymbol{x}$-axis does not induce any change of the internal state of the mechanism if the parameter $\gamma$ is null. In order to calculate the global Jacobian matrix $\mathbf{J}$ and to perform the sensitivity and observability studies, 231 measurement points are taken over the entire workspace. Figure 11 shows the variation of the TCP position as a consequence of a variation on each parameter successively, i.e., $\sigma_{P_{j}}$. The ranges of parameter variation are $0.1 \mathrm{~mm}, 1^{\circ}$ and $1000 \mathrm{~N} / \mathrm{mm}$ for the parameters of length, angle, and stiffness, respectively. For the sake of simplicity the influence of an error of the guide angle $\gamma$ is not represented. A variation of $1^{\circ}$ of this parameter, corresponding to the variation range, induces a variation of more than $7 \mathrm{~mm}$ on the TCP position. This would render the other variations not visible on the graph. The angle $\gamma$ is the most influent parameter for both redundant and non-redundant mechanisms. The influence of elasticity parameters $\left(k_{r a 1}\right.$ to $\left.k_{r a 3}\right)$ on the TCP position is null because the geometrical parameters are not modified when the study of the influence of these parameters is carried out. For the parameters belonging to both mechanisms, cf. section 2.3, it appears clearly that the parameter variations are more influent (about 25\%). The calibration of the non-redundant PKM will then tend to be more stable and accurate. This figure illustrates also the fact that redundant PKMs are more robust to parameter errors.

Concerning the observability studies achieved for both redundant and nonredundant mechanisms, all influent parameters are identifiable. For the redundant and non-redundant mechanisms, the condition numbers of the Jacobian 


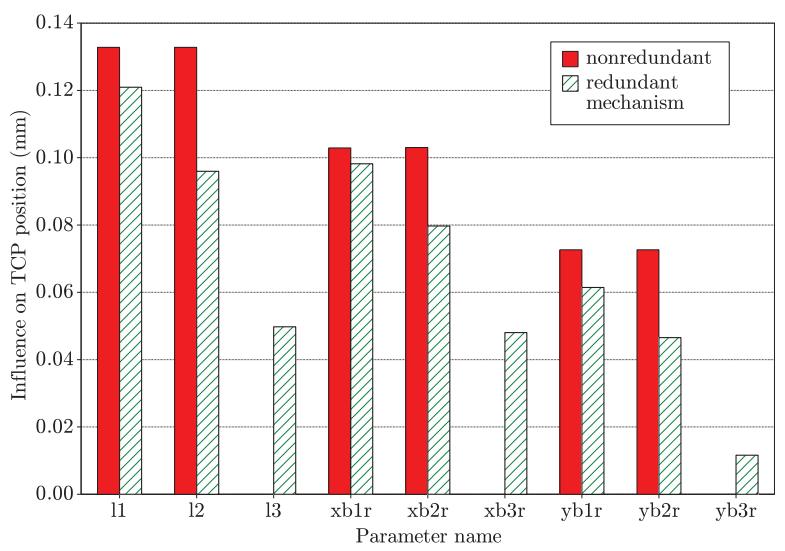

Figure 11. Sensitivity analysis.

matrix are $c_{\mathrm{r}}=5845$ and $c_{\mathrm{nr}}=3160$, respectively. The angle parameter $\gamma$ can be advantageously determined from a separated calibration process by an equivalent movement of all actuators in order to improve the observability of the calibration process. The new condition numbers without the parameter $\gamma$ are $c_{\mathrm{r}}=773$ and $c_{\mathrm{nr}}=60$. This presumes that the non-redundant mechanism will be more stable as far as the optimization process is concerned. However, this result can probably be more attributed to the fact that the number of parameters is smaller in the second case.

\subsection{Identification}

The cost-function that is to be minimized during the calibration process is expressed as a least-squares problem as

$$
f=\sum_{i=1}^{m}\left(\boldsymbol{X}_{\text {meas }}^{i}-\boldsymbol{X}_{\text {fegm }}^{i}\right)^{T}\left(\boldsymbol{X}_{\text {meas }}^{i}-\boldsymbol{X}_{\text {fegm }}^{i}\right)
$$

where $\boldsymbol{X}_{\text {meas }}^{i}$ is the measured situation of the platform for the PKM configuration $i$ and $\boldsymbol{X}_{\mathrm{fegm}}^{i}$ is the corresponding platform situation calculated through the elasto-geometrical model with the nominal parameters $\boldsymbol{\xi}_{\text {nom }}$.

Simulations were then carried out for the Redundant Triglide. A number of 9 parameters could be included into the identification step: $l_{1}, l_{2}, l_{3}, x_{b 1 r}$, $y_{b 1 r}, x_{b 2 r}, y_{b 2 r}, x_{b 3 r}, y_{b 3 r}$, whereas 6 parameters were identified for the nonredundant mechanism: $l_{1}, l_{2}, x_{b 1 r}, y_{b 1 r}, x_{b 2 r}, y_{b 2 r}$. Identification was done by using a non-linear optimization, a trust-region reflective Newton algorithm integrated in the Isqnonlin function from Matlab ${ }^{\mathrm{TM}}$. The simulated measured TCP positions along the $\boldsymbol{x}$ and $\boldsymbol{y}$-directions were obtained through the forward elasto-geometrical model with the real geometrical/stiffness parameters $\boldsymbol{\xi}$.

Figure 12(a) shows the mean final parameter error for the calibration of both 
mechanisms with respect to the number of measurement points (a Gaussian noise with a standard deviation of $10 \mu \mathrm{m}$ is added to each measurement point). As expected, the calibration results for the non-redundant mechanism are better than the results obtained with the Redundant Triglide. The mean TCP positioning accuracy was calculated for a set of 10 points taken within the workspace. Figure 12(b) shows the resulting Cartesian error for the two identified models with respect to the number of measurement points. These simulation results show that the proposed elasto-geometrical method is efficient and can be involved for the calibration of redundant PKMs with slender links.

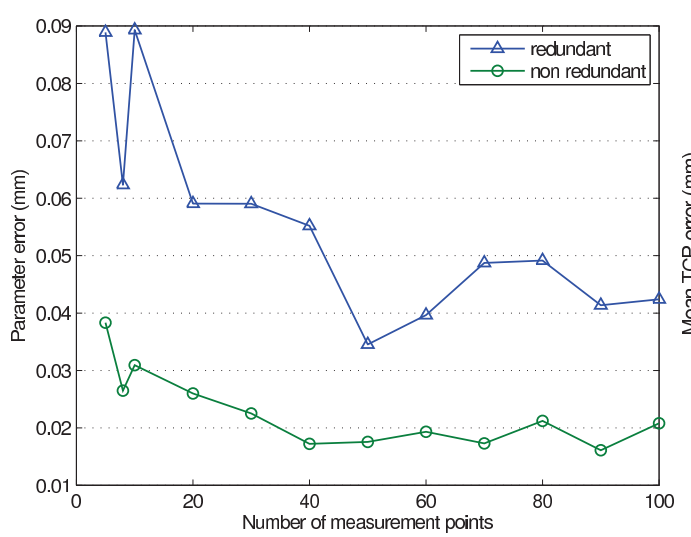

(a)

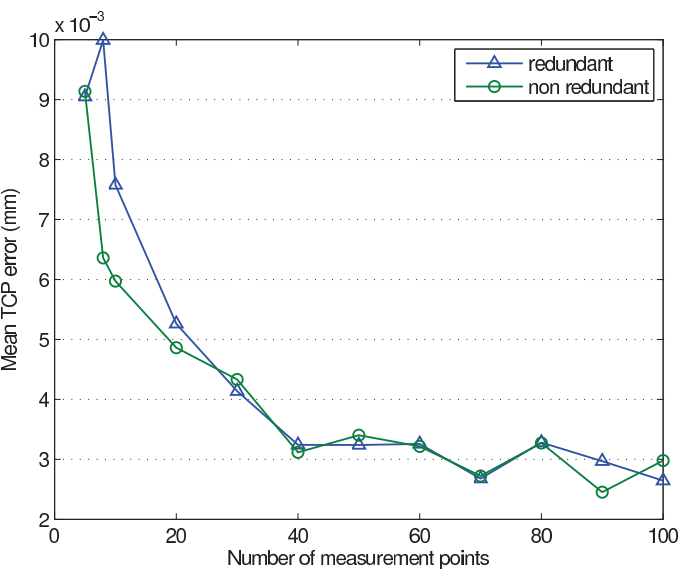

(b)

Figure 12. Parameter error and positioning error with respect to the number of measurement points.

\section{Experimental Validation}

\subsection{Description and elasto-geometrical modeling of the Scissors-Kinematics machine}

The method that has been proposed for the elasto-geometrical modeling of redundant PKMs has been tested on the Scissors-Kinematics machine developed at the Fraunhofer Institute for Machine Tools and Forming Technology IWU in Chemnitz for tool and die machining [27]. As shown in Fig. 13, the structure of this machine includes a parallel mechanism with a second order of redundancy that can be considered as planar. The elasto-geometrical modeling method that has been developed so far involves mechanisms with one redundant branch. However, its application is also suitable for mechanisms with a higher order of redundancy and, therefore, it has been applied to the Scissors-Kinematics architecture.

The Scissors-Kinematics redundant parallel structure includes a moving plat- 


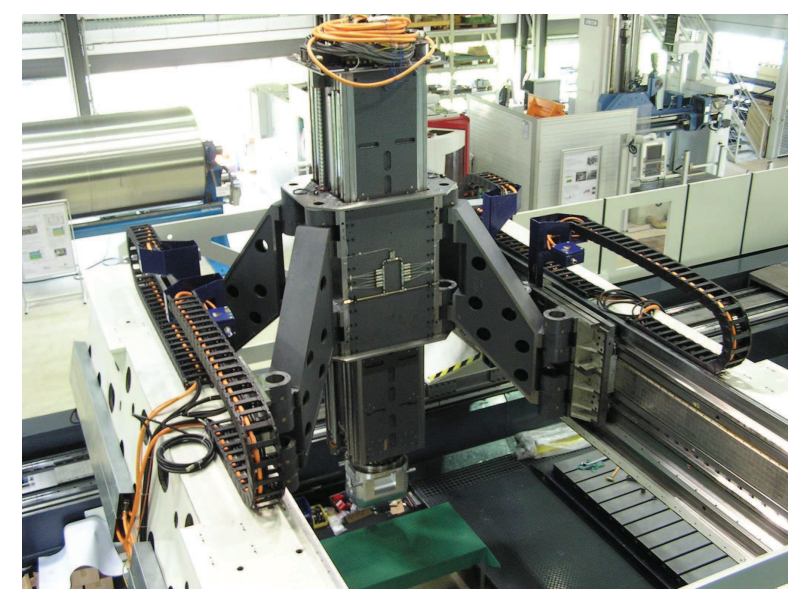

Figure 13. The Scissors-Kinematics.

form, four linear actuators along the $\boldsymbol{y}$-axis and five fixed-length rods (Fig. 14(a)). The vector of parameters $\boldsymbol{\xi}$ which has been used to derive the elasto-geometrical models includes (Fig. 14(b)):

- $l_{\mathrm{i}}$ that is the length of $\operatorname{rod} i$,

- $x_{\text {air }}, y_{\text {air }}$ that give the relative position of the platform joint $A_{i}$ within the platform frame,

- $x_{\text {bir }}, y_{\text {bir }}$ that give the relative position of the carrier joint $B_{i}$ in the frame attached to the carrier $i$,

- $d_{1}, d_{2}$ and $\gamma_{1}, \gamma_{2}$ that describe respectively the guide positions and orientation with respect to the reference frame,

- $k_{i}$ that is the radial stiffness coefficient given for the joint at node $B_{i}$ and which represents the stiffness of $\operatorname{rod} i$ and both joints linked to it.

In order to give an idea of the machine size, the nominal values of those parameters are gathered in Table 2 .

Table 2

Scissors' nominal parameter values ( $\mathrm{mm}$, deg and $\mathrm{kN} / \mathrm{mm}$ ).

\begin{tabular}{|c|c|c|c|c|c|c|c|c|}
\hline$l_{\mathrm{i}}$ & $x_{\text {air }}$ & $y_{\text {air }}$ & $x_{\text {bir }}$ & $y_{\text {bir }}$ & $d_{\mathrm{i}}$ & $\gamma_{\mathrm{i}}$ & $k_{1} \ldots k_{4}$ & $k_{5}$ \\
\hline 950 & 520 & 280 & 310 & 80 & 1460 & 0 & 500 & 250 \\
\hline
\end{tabular}

\subsection{Elasto-Geometrical Modeling Validation}

For several TCP positions along the $\boldsymbol{x}$-axis a variation was applied on one of the parameters, the new actuator positions were calculated with this modified parameter set and all actuators were driven to these positions. The TCP displacement was then measured along the $\boldsymbol{x}$ - and $\boldsymbol{y}$-axes with the KGM 182 grid-encoder from Heidenhain, presented in Fig. 15. The grid-encoder is a twodimensional contact-free measurement system with a sub-micron measurement 


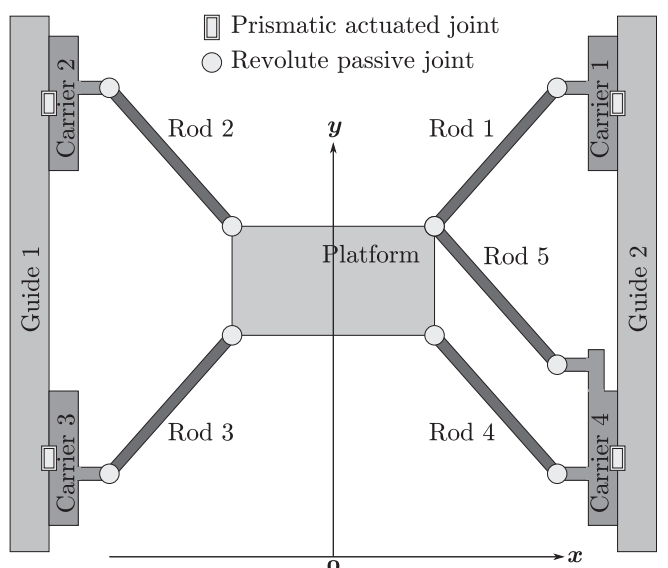

(a)

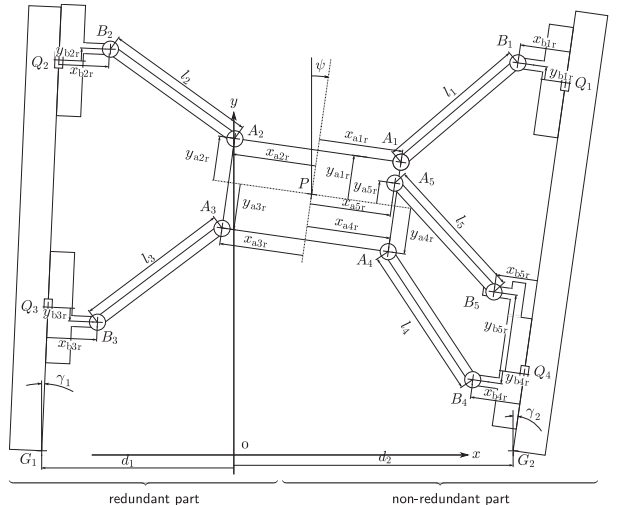

(b)

Figure 14. Simplified view (a) and geometrical parameters (b) of the Scissors-Kinematics

accuracy. The scanning head is mounted directly in the spindle and the plate is fixed on the machine table. The measurement results are presented in Fig. 16 for a variation of $30 \mu \mathrm{m}$ on parameter $l_{1}$ and compared to the results obtained with the elasto-geometrical modeling method.
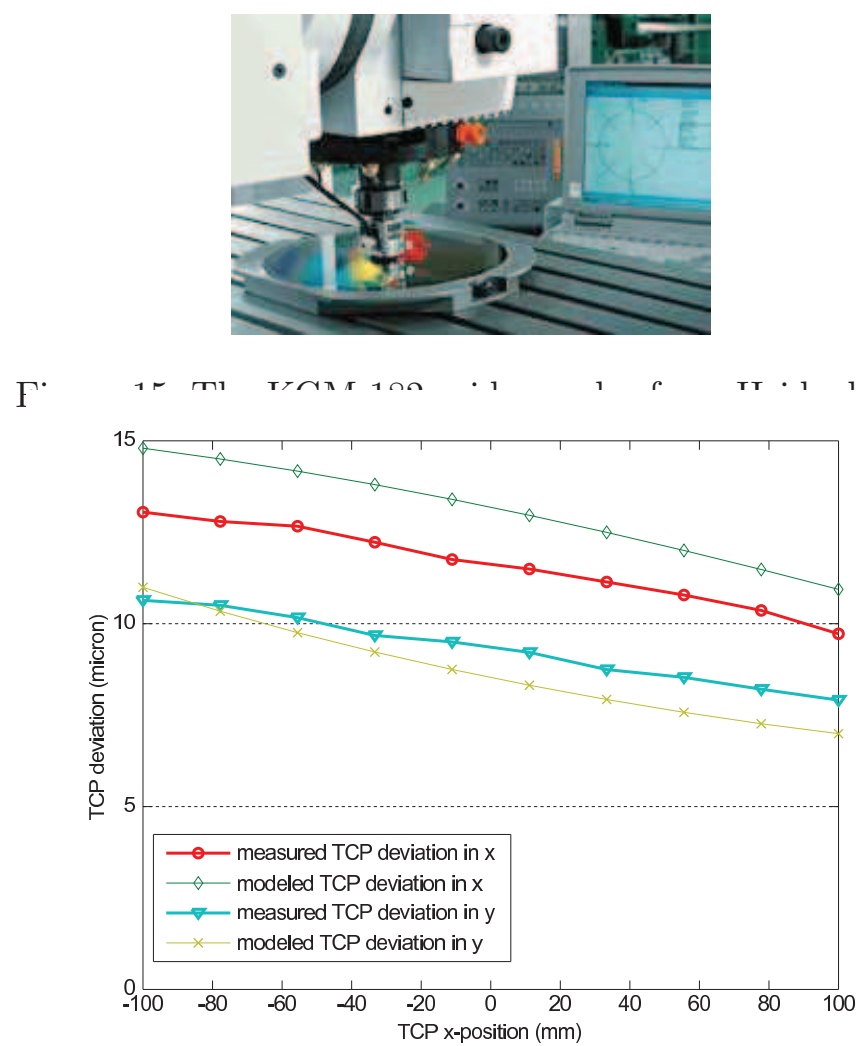

Figure 16. Elasto-geometrical modeling validation for parameter $l_{1}$.

Figure 17 presents the comparison of TCP deviations between the experiments and the values obtained with the elasto-geometrical model for the validation 
of the elasto-geometrical modeling method. It shows the mean TCP deviation over a movement along the $\boldsymbol{x}$-axis when a variation of $30 \mu \mathrm{m}$ is imposed on all parameters successively $\left(10^{-5} \mathrm{rad}\right.$ for the parameters $\gamma_{1}$ and $\left.\gamma_{2}\right)$. It can be clearly seen that the TCP variation is far smaller than the parameter errors. The TCP position is barely sensitive to parameter variations.

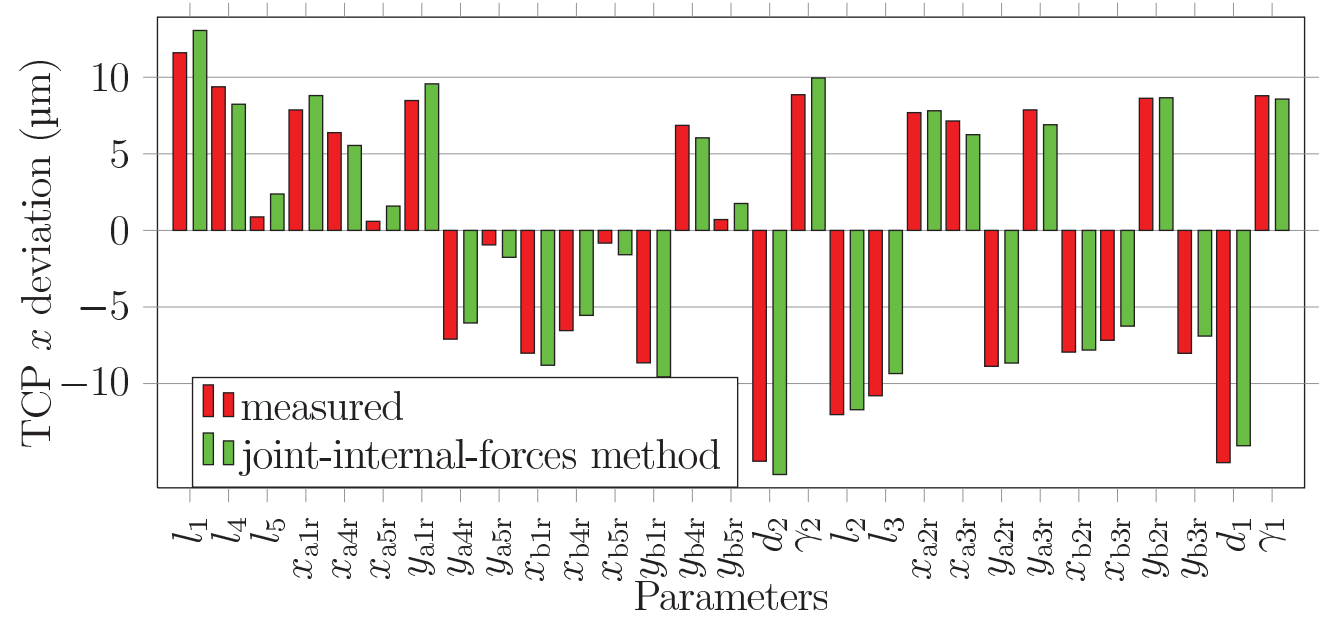

Figure 17. Deviations on the TCP $x$ position.

The modeled TCP displacements due to variations in the stiffness parameters $k_{1}$ to $k_{5}$ cannot be obtained with this method since they only have an influence in a constrained mechanism. Thus, a sensitivity analysis was carried out with a constrained displacement of $-50 \mu \mathrm{m}$ on $q_{1}$ and $+50 \mu \mathrm{m}$ on $q_{4}$. A variation of $10 \mathrm{kN} / \mathrm{mm}$ was then applied on the stiffness parameters and the TCP displacement was computed. The mean TCP displacement over the whole workspace is presented in Fig. 18. The TCP displacements are too small to be measurable.

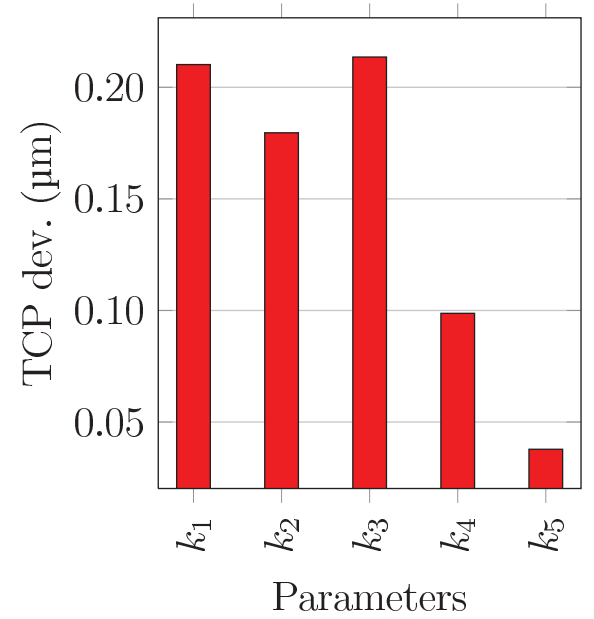

Figure 18. Sensitivity analysis for stiffness parameters.

The average deviation between the measured TCP displacements and those obtained by the elasto-geometrical model for all parameters and over the whole 
$\boldsymbol{x}$-movement is $14 \%$. As a comparison, the geometrical model has a deviation of $114 \%$ for the parameters of the non-redundant part containing rods 1,4 , and 5. For the redundant part, it is $100 \%$ because these parameters are not taken into account in the model. The elasto-geometrical modeling is suitable for reproducing the machine behavior against parameter errors. It can then be used in a calibration process.

\subsection{Elasto-geometrical calibration validation}

The calibration of the Scissors-Kinematics was carried out using the gridencoder to measure 80 TCP positions over the whole workspace. All drives were position-controlled during the measurement. The position control on all actuators could only be achieved by recording the positions of the redundant actuators beforehand during a force-free workspace mapping. For this calibration nine parameters were included in the optimization process $\left(\gamma_{1}, \gamma_{2}, x_{\mathrm{a} 1 \mathrm{r}}\right.$, $\left.l_{3}, x_{\mathrm{a} 2 \mathrm{r}}, y_{\mathrm{a} 1 \mathrm{r}}, l_{4}, y_{\mathrm{a} 2 \mathrm{r}}, y_{\mathrm{a} 4 \mathrm{r}}\right)$. Although the parameters $d_{1}$ and $d_{2}$ have the largest sensitivity, they do not appear in the list of parameters to be identified because of the column normalization of the Jacobian matrix used during the optimization process. The stiffness parameters cannot be included into the calibration process since the influence of a variation in these parameters on the TCP position cannot be detected by the measuring device. The value of the excluded parameters remains nominal during the calibration process. The condition number of the normalized Jacobian matrix is then 280 . The calibration optimization process converges quickly. The optimization criterion before the first iteration is $1.308 \mathrm{~mm}^{2}$; its final value is $0.00365 \mathrm{~mm}^{2}$.

The calibration results were then tested by using a force control in the redundant drives in order to reduce the risk of over-constraints in the mechanism. The mean position difference for the redundant actuators between the results of the forward geometrical model with the calibrated parameters and the measurements are $\Delta q_{2}=0.873 \mathrm{~mm}$ and $\Delta q_{3}=0.305 \mathrm{~mm}$ for actuators 2 and 3 , respectively. The calibrated parameters did not allow for switching to the full position control because the position difference is too large. The same measurement data were involved in the calibration with a geometrical model. An average error of $10 \mu \mathrm{m}$ was obtained during a $100 \mathrm{~mm}$-radius circular test with the optimized parameters. The measurement data are then excluded as being the cause of the discrepancy between the results of the elasto-geometrical calibration and the real mechanism.

The Scissors-Kinematics is a very stiff mechanism, which is certainly a great quality, but, as could be seen from the validation test of the elasto-geometrical modeling method, the influence of parameter errors is measured with difficulty. Thus, the elasto-geometrical method combined with the intrinsic measurement 
errors may be less adaptable to a great stiffness. Another problem, which is intrinsic to redundantly actuated PKMs, is that more parameters are needed to describe the mechanism; the degree of freedom of the end-effector is, however, constant. This means that the ratio between the degree of sensing and the number of parameters is smaller. A possible solution for the calibration of such mechanisms could be the use of extra sensors to augment the degree of sensing.

\section{Conclusion}

In this paper a method was developed for the calculation of the TCP Cartesian position for redundant parallel kinematic mechanisms that takes into account the mechanism's own weight, the external forces and the internal constraints linked to the actuation redundancy. The method uses a partly analytical finiteelement analysis based on beam elements, so that it is quick enough for an on-line implementation. The method can generally be applied to all kinds of redundant PKMs with one or more redundant actuators. An example of application is given for a planar redundant mechanism with one redundant actuated branch. This method can also be applied for the study of the influence of machine parameter errors on the TCP, thus it is adapted for the calibration of such mechanisms. The calibration simulations revealed the complementary facts that redundant PKMs are more robust to parameter errors and that for this reason they are more difficult to calibrate than the classical non-redundant PKMs. This difficulty suggests the development of new calibration methods for redundant PKMs. For instance, a self-calibration strategy could be used where the redundant actuators would act as extra measuring systems or extra sensors could be used on passive joints. The experimental results confirmed these two facts: the modeling is suitable to represent the influence of parameter errors and the redundantly actuated mechanisms are more difficult to calibrate because of the influence of internal constraints.

\section{References}

[1] M. Valášek, V. Bauma, Z. Šika, T. Vampola, Redundantly actuated parallel structures - principle, examples, advantages, in: Proceedings of the 3rd Chemnitz Parallel Kinematics Seminar, 2002, pp. 993-1009.

[2] H. Liao, T. Li, X. Tang, Singularity analysis of redundant parallel manipulators, in: IEEE International Conference on Systems, Man and Cybernetics, 2004, pp. 4214-4220. 
[3] F. Marquet, Robots parallèles et redondance, Ph.D. thesis, Université Montpellier II (2002).

[4] S. Kock, W. Schumacher, A parallel x-y manipulator with actuation redundancy for high-speed and active-stiffness applications, in: Proceedings of the 1998 IEEE International Conference on Robotics \& Automation, 1998, pp. 22952300 .

[5] S. H. Lee, J. H. Lee, B.-J. Yi, S. H. Kim, Y. K. Kwak, Optimization and experimental verification for the antagonistic stiffness in redundantly actuated mechanisms: a five-bar example, Mechatronics 15 (2005) 213-238.

[6] A. Müller, Internal preload control of redundantly actuated parallel manipulators backlash avoiding control, in: Proceedings of the IEEE International Conference on Robotics and Automation, 2005, pp. 960-965.

[7] A. J. Patel, K. F. Ehmann, Calibration of a hexapod machine tool using a redundant leg, International Journal of Machine Tools \& Manufacture, Vol. 40(4) (2000) 489-512.

[8] G. Ecorchard, R. Neugebauer, P. Maurine, Self-calibration of a redundantly actuated parallel kinematic machine tool, in: Proceedings of the 5th Chemnitz Parallel Kinematics Seminar, 2006, pp. 477-496.

[9] D. Daney, Kinematic calibration of the Gough platform, Robotica, Vol. 21 (2003) 677-690.

[10] D. Chakarov, Study of the antagonistic stiffness of parallel manipulators with actuation redundancy, Mechanism and Machine Theory, Vol. 39(6) (2004) 583601.

[11] H. Cheng, Y.-K. Yiu, Z. Li, Dynamics and control of redundantly actuated parallel manipulators, IEEE/ASME Transactions on Mechatronics, Vol. 8, No. 4 (2003) 483-491.

[12] R. Neugebauer, Parallelkinematische Maschinen, Springer-Verlag, 2006.

[13] J. I. Jeong, D. Kang, Y. M. Cho, J. Kim, Kinematic calibration for redundantly actuated parallel mechanisms, Journal of Mechanical Design (2004) 307-318.

[14] D. Deblaise, X. Hernot, P. Maurine, A systematic analytical method for PKM stiffness matrix calculation, in: Proceedings of the IEEE International Conference on Robotics and Automation, 2006, pp. 4213-4219.

[15] G. Ecorchard, P. Maurine, Self-calibration of delta parallel robots with elastic deformation compensation, in: Proceedings of the IEEE/RSJ International Conference on Intelligent Robots and Systems, 2005, pp. 1283-1288.

[16] F. Marquet, F. Pierrot, O. Company, A statistical approach for the computation of the forward kinematic model of redundantly actuated mechanisms, in: IEEE International Conference on Intelligent Robots and Systems, 2003, pp. 35583564 . 
[17] A. Pashkevich, D. Chablat, P. Wenger, Stiffness analysis of overconstrained parallel manipulators, Mechanism and Machine Theory 44 (2009) 966-982.

[18] T. Huang, X. Zhao, D. Whitehouse, Stiffness estimation of a tripod-based parallel kinematic machine, IEEE Transactions on Robotics and Automation 18(1) (2002) 50-58.

[19] Z. Bi, W. Gruver, W. Zhang, S. Lang, Automated modeling of modular robotic configurations, Robotics and Autonomous Systems (2006) 1015-1025.

[20] U. Meißner, A. Menzel, Die Methode der finiten Elemente, Springer-Verlag, 1989.

[21] W.-K. Yoon, T. Suehiro, Y. Tsumaki, M. Uchiyama, Stiffness analysis and design of a compact modified delta parallel mechanism, Robotica, Vol. 22(4) (2004) 463-475.

[22] B. Klein, FEM, Grundlagen und Anwendungen der Finite-Element-Methode, Vieweg, 2003.

[23] C. Lawson, R. Hanson, Solving Least Squares Problems, Society for Industrial and Applied Mathematics, 1974.

[24] A. Nahvi, J. Hollerbach, V. Hayward, Calibration of a parallel robot using multiple kinematic closed loops, in: Proceedings of the International Conference on Robotics and Automation, San Diego, Vol.1, 1994, pp. 142-148.

[25] W. Khalil, J. Caenen, C. Enguehard, Identification and calibration of robots, in: First International Symposium on Experimental Robot, Montreal, Canada, 1989.

[26] S. Besnard, W. Khalil, Identifiable parameters for parallel robots kinematic calibration, in: Proceedings of the IEEE International Conference on Robotics and Automation, 2001, pp. 2859-2866.

[27] R. Neugebauer, S. Ihlenfeldt, J. Kirchner, F. Adam, Redundante hybridkinematik für den großwerkzeug- und formenbau, in: Tagungsband den 2. Parallel Kinematik Seminar, 2000, pp. 153-168. 